\title{
Fiberized Diamond-Based Vector Magnetometers
}

\author{
Georgios Chatzidrosos $^{1,2 *}$, Joseph Shaji Rebeirro ${ }^{1,2}$, Huijie Zheng ${ }^{1,2 *}$, Muhib Omar ${ }^{1,2}$, \\ Andreas Brenneis ${ }^{3}$, Felix M. Stürner ${ }^{3}$, Tino Fuchs ${ }^{3}$, Thomas Buck ${ }^{3}$, Robert Rölver ${ }^{3}$, \\ Tim Schneemann ${ }^{1,2}$, Peter Blümler ${ }^{2}$, Dmitry Budker ${ }^{1,2,4}$ and Arne Wickenbrock ${ }^{1,2}$
}

${ }^{1}$ Helmholtz-Institut, GSI Helmholtzzentrum für Schwerionenforschung, Mainz, Germany, ${ }^{2}$ Department of Physics, Johannes Gutenberg-Universität Mainz, Mainz, Germany, ${ }^{3}$ Corporate Sector Research and Advance Engineering, Robert Bosch GmbH, Renningen, Germany, ${ }^{4}$ Department of Physics, University of California, Berkeley, Berkeley, CA, United States

We present two fiberized vector magnetic-field sensors, based on nitrogen-vacancy (NV) centers in diamond. The sensors feature sub-nT/ $\sqrt{\mathrm{Hz}}$ magnetic sensitivity. We use commercially available components to construct sensors with a small sensor size, high photon collection, and minimal sensor-sample distance. Both sensors are located at the end of optical fibres with the sensor-head freely accessible and robust under movement. These features make them ideal for mapping magnetic fields with high sensitivity and spatial resolution $(\leq \mathrm{mm})$. As a demonstration we use one of the sensors to map the vector magnetic field inside the bore of $a \geq 100 \mathrm{mT}$ Halbach array. The vector field sensing protocol translates microwave spectroscopy data addressing all diamonds axes and including double quantum transitions to a 3D magnetic field vector.

Keywords: NV, nitrogen-vacancy, magnetomerters, vector magnetometry, quantum sensing, fiberized system, nitrogen-vacancy (NV) center

\section{INTRODUCTION}

Nitrogen-vacancy (NV) centers in diamond have attracted attention as magnetic field sensors with high spatial resolution (Balasubramanian et al., 2008; Maze et al., 2008; Rittweger et al., 2009) and sensitivity (Barry et al., 2016; Chatzidrosos et al., 2017; Zhang et al., 2021). Their sensitivity has been demonstrated to be as low as sub-pT level, for AC fields (Wolf et al., 2015) and with hybrid magnetometers at the low-frequency regime (Xie et al., 2021) The range of their application includes, but is not limited to, single neuron-action potential detection (Barry et al., 2016), single protein spectroscopy (Lovchinsky et al., 2016), as well as in vivo thermometry (Kucsko et al., 2013). Advantages of NV-based magnetometers, compared to other magnetic field sensors, include their ability to operate in wide temperature and magnetic field ranges (Zheng et al., 2017). The ability to operate them also without the use of microwaves (Wickenbrock et al., 2016; Zheng et al., 2017; Zheng et al., 2020), has recently enabled a variety of new applications in environments where microwaves (MW) would be detrimental (Chatzidrosos et al., 2019).

The NV consists of a substitutional nitrogen and an adjacent vacant carbon site. It can appear in different orientations along the crystallographic axes of the diamond lattice. This enables vector measurements of magnetic fields (Clevenson et al., 2018). Vector magnetometry itself can be useful in magnetic navigation applications (Cochrane et al., 2016), magnetic anomaly detection, current and position sensing, and the measurement of biological magnetic fields (Barry et al., 2016). Vector measurements near a background field of $\sim 100 \mathrm{mT}$, where the NV's ground state level anti-crossing (GSLAC) occurs, are of particular interest (Zheng et al., 2020). Some of the challenges of vector measurements near the GSLAC field include the necessity to precisely align the NV and the bias 
magnetic field axes for optimum sensitivity of the microwave-free method (Zheng et al., 2017) or the need to account for transversal field related nonlinearities of the NV gyromagnetic ratio when performing microwave spectroscopy.

One of the challenges NV magnetometers face is their low photon-collection efficiency. Approaches to increase the efficiency include, use of solid immersion lenses (Hadden et al., 2010; Siyushev et al., 2010; Sage et al., 2012), or employment of infrared absorption(Acosta et al., 2010; Dumeige et al., 2013; Jensen et al., 2014; Chatzidrosos et al., 2017; Dumeige et al., 2019). Photoluminescence (PL) for fiberized sensors is preferentially collected with the same fiber delivering the pump light but detected on the input side of the fiber (Patel et al., 2020). Despite considerable effort, even modern sensors typically just feature a PL-to-pump-light ratio of about 0.1\% (Barry et al., 2016; Patel et al., 2020).

In this paper, we discuss the construction of a fiberized NV-based vector magnetic field sensors and compare it with another sensor of the same kind. One sensor is constructed in Helmholtz Institute Mainz, referred to as Mainz sensor in the following and the other one is constructed by Robert Bosch $\mathrm{GmbH}$, referred to as Bosch sensor in the following. They both achieve sub $\mathrm{nT} / \sqrt{\mathrm{Hz}}$ magnetic sensitivity. The sensor constructed in Mainz achieved a sensitivity of $0.45 \mathrm{nT} /$ $\sqrt{\mathrm{Hz}}$, limited by intensity noise of the pump laser, with $11 \mathrm{pT} / \sqrt{\mathrm{Hz}}$ photon-shot noise sensitivity and $0.5 \%$ PL-to-pump power ratio taking the fiber coupling efficiency into account. The sensor constructed by Bosch achieved a sensitivity of $0.34 \mathrm{nT} / \sqrt{\mathrm{Hz}}$, which was approximately one order of magnitude larger than the expected photon-shot noise limited sensitivity (Stürner et al., 2021). The PL-to-pump power ratio was $0.3 \%$ taking the fiber-coupling efficiency into account. The components used for the construction of both sensors are commercially available. They allow for a small sensor size $\left(11 \times 40 \mathrm{~mm}^{2}\right.$ for Mainz and $15 \times 25 \mathrm{~mm}^{2}$ for Bosch $)$ with maximized PL-to-pump-light ratio, as well as robustness to movement, which also makes the sensors portable. All of this makes the sensors ideal for mapping magnetic fields and measuring in regions that are not easily accessible. The magnetometers are constructed in such a way that they allow close proximity of the sensors to magnetic field sources, thus allowing for high spatial resolution when mapping magnetic fields. After demonstrating the sensitivity of the sensors and explaining the principles of vector magnetometry with NV centers, we used the Mainz sensor to perform spatially resolved optically detected magnetic resonance (ODMR) measurements covering an area of $\times 2030 \mathrm{~mm}^{2}$ inside a Halbach-magnet array. The Halbach array itself provides a highly homogeneous magnetic field around $100 \mathrm{mT}$ which makes it ideal for near-GSLAC magnetic field measurements and studies with $\mathrm{NV}$ centers. We present the analysis for the translation of the extracted frequency measurements into magnetic field. Details about the construction of the highly homogeneous Halbach array is subject of another publication (Wickenbrock et al., 2020).

\section{EXPERIMENTAL SETUP}

The diamond sample used for the Mainz sensor is a $2.0 \times 2.0 \times$ $0.5 \mathrm{~mm}^{3}$ type Ib (100)-cut, high-pressure high-temperature
(HPHT) grown sample, purchased from Element Six. The initial [N] concentration of the sample was specified as < $10 \mathrm{ppm}$. The sample was irradiated with $5 \mathrm{MeV}$ electrons at a dose of $2 \times 10^{19} \mathrm{~cm}^{-2}$ and then annealed at $700^{\circ} \mathrm{C}$ for $8 \mathrm{~h}$. The diamond sample used for the Bosch sensor is a $0.8 \times 0.8 \times$ $0.5 \mathrm{~mm}^{3}$ (111)-cut, $99.97 \%{ }^{12} \mathrm{C}$ enriched, HPHT grown diamond. The sample was irradiated with $2 \mathrm{MeV}$ electrons at a dose of $2 \times$ $10^{18} \mathrm{~cm}^{-2}$ at room temperature and then annealed at $1,000^{\circ} \mathrm{C}$ for $2 \mathrm{~h}$ in vacuum. The $\left[\mathrm{NV}^{-}\right]$concentration was determined by electron spin resonance to be 0.4 ppm (Stürner et al., 2021).

Figure 1A shows the relevant energy levels of the negatively charged NV center, which we use for magnetometry. The ground and excited spin-triplet states of the NV are labeled ${ }^{3} \mathrm{~A}_{2}$ and ${ }^{3} \mathrm{E}$, respectively [Figure 1A] , the transition between them has a zerophonon line at $637 \mathrm{~nm}$, but can be exited by more energetic photons (of shorter wavelengths) due to phonon excitation in the diamond lattice. The lower and upper electronic singlet states are ${ }^{1} \mathrm{E}$ and ${ }^{1} \mathrm{~A}_{1}$, respectively. While optical transition rates are spinindependent, the probability of nonradiative intersystem crossing from ${ }^{3} \mathrm{E}$ to the singlets is several times higher (Dumeige et al., 2013) for $m_{s}= \pm 1$ than that for $m_{s}=0$. As a consequence, under continuous illumination with green pump light $(532 \mathrm{~nm}), \mathrm{NV}$ centers accumulate mostly in the ${ }^{3} \mathrm{~A}_{2}, m_{s}=0$ ground state sublevel and in the metastable ${ }^{1} \mathrm{E}$ singlet state. For metrology applications, the spins in the ${ }^{3} \mathrm{~A}_{2}$ ground state can be coherently manipulated with microwave fields.

Figure 1B shows the experimental setup used for the measurements conducted in Mainz. To initialize the NV centers to the ground-triplet state, we use a $532 \mathrm{~nm}$ (green) laser (Laser Quantum, gem532). To reduce intensity noise from the laser source, the light intensity is stabilized using an acousto-optic modulator (AOM, ISOMET-1260C with an ISOMET 630C-350 driver) controlled through a proportionalintegral-derivative controller (PID, SIM960), in a feedback loop. The green laser is normally stabilized to a laser power of $200 \mathrm{~mW}$. The green laser is then coupled into a $2 \mathrm{~m}$ long, high-power multi-mode fiber cable (Thorlabs, MHP365L02).

The MW to manipulate the NV spins are generated with a MW function generator (FG; SRS, SG394). They are amplified with a $16 \mathrm{~W}$ amplifier (Amp; Mini-Circuits, ZHL-16W-43+) and passed through a circulator (Mini-Circuits, CS-3.000) before they are applied to the NV centers using a $\mathrm{mm}$-sized wire loop. The other side of the wire is directly connected to ground. The radius of the wire used for the wire loop is $50 \mu \mathrm{m}$. The wire is then attached to the optical fiber allowing the two parts to move together. For the magnetic resonance measurements presented in this paper, the MW frequency was scanned between $3,800 \mathrm{MHz}$ and 5,000 MHz. Information on the experimental setup used to conduct the measurements with the Bosch sensor can be found in Ref (Stürner et al., 2021). The data presented for the Bosch sensor are acquired at $20 \mathrm{~mW}$ of pump-light power and $0.06 \mathrm{~mW}$ of MW power.

A schematic of the Mainz fiberized magnetic field sensor head is shown in Figure 1C. The diamond is glued to a parabolic condenser lens, which itself is glued onto a $11 \mathrm{~mm}$ focal length lens and attached to a fiber collimator (Thorlabs, F220SMA-532) to which the high-power multi-mode fiber (Thorlabs, 
A

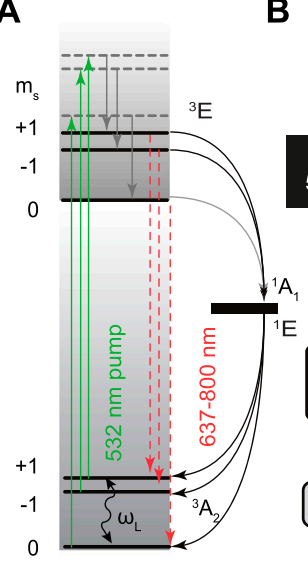

B

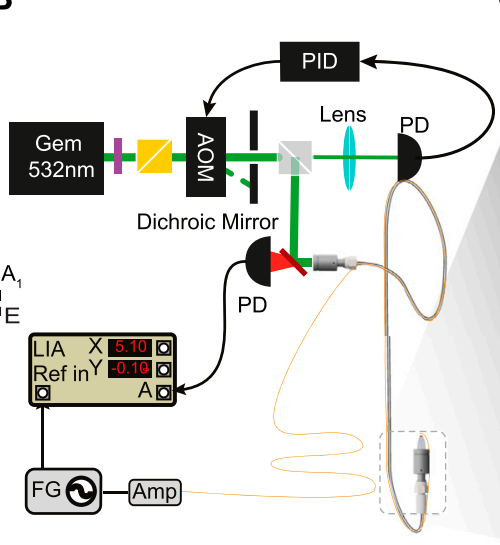

C

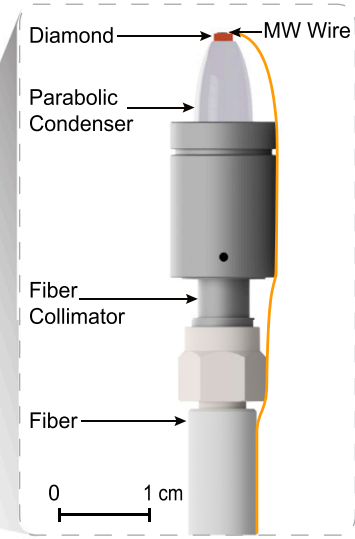

D

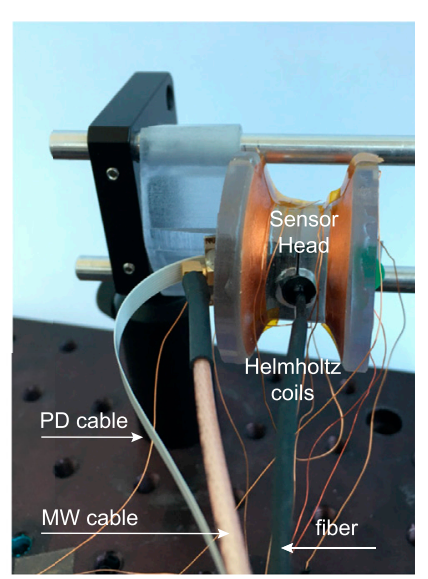

FIGURE 1 | (A) Relevant energy levels of the NV center. The states as well as their spin projection $\mathrm{m}_{s}$ are labeled. Solid green lines represent pump light, solid gray lines phononic de-excitation, dashed red lines represent PL, curved gray lines represent inter-system crossing. (B) Experimental setup in Mainz (the experimental setup by Bosch can be found in Ref(Stürner et al., 2021)). (C) Schematic of the fiberized sensor head. (D) Photograph of the fiberized sensor by Robert Bosch GmbH. The abbreviations used in the figure are; PD: Photo-diode, AOM: acousto-optic modulator, PID: proportional-integral-derivative controller, LIA: lock-in amplifier, FG: function generator, Amp: amplifier, MW: microwave.

MHP365L02) is connected. The MW wire loop is attached to the other side of the diamond to provide the rapidly oscillating magnetic fields required for this magnetic field detection scheme. The light is delivered to the diamond via the parabolic condenser, lens, collimator and the high-power fiber. The same components collect the spin-state-dependent red PL of the NV ensemble. On the other side of the fibre the PL is filtered using a longpass dichroic filter (Thorlabs, DMLP605) which is also used to couple the incoming green light into the fiber. After the dichroic filter residual reflected green light is removed by a notch filter (Thorlabs, NF533-17) and the PL is focused onto a photodiode (PD; Thorlabs, PDA36a2). The detected signal with the PD is connected to a lock-in amplifier (LIA; SRS, SR830). With this setup we were able to achieve $0.5 \%$ PL-to-pump-light ratio, which is an order-of-magnitude improvement compared to other fiberized sensors (Patel et al., 2020). A photograph of the Bosch sensor can be seen in Figure 1D. The sensor head, containing a microwave resonator, a custom designed balanced photodetector, and the diamond, which was glued to the collimated output of a single-mode fiber, is located inside a custom designed Helmholtz coil. The Helmholtz coil was used to generate a magnetic bias field of $1.07 \mathrm{mT}$ and the collimation of the laser beam was achieved with a gradient-index lens (GRIN). Further details on the setup used for the Bosch sensor can be found in Ref. (Stürner et al., 2021). The final sensor head, of the Mainz sensor, has a diameter of $\leq 11 \mathrm{~mm}$ and a height of $40 \mathrm{~mm}$, in a configuration that allows for $\leq 300 \mu \mathrm{m}$ average distance between sample and sensor, these dimensions refer to not just the NV-containing diamond but to the overall sensor-head footprint, including parabolic lens and fiber collimator. The $2 \mathrm{~m}$ long fiber with the attached MW wire and the fiberized sensor head for the Mainz sensor (shown in Figure 1C) can be moved independently of the other components. The footprint of the sensor head of the Bosch sensor was $15 \times 25 \mathrm{~mm}^{2}$, with these dimensions referring to the sensor-head as well. The smallest

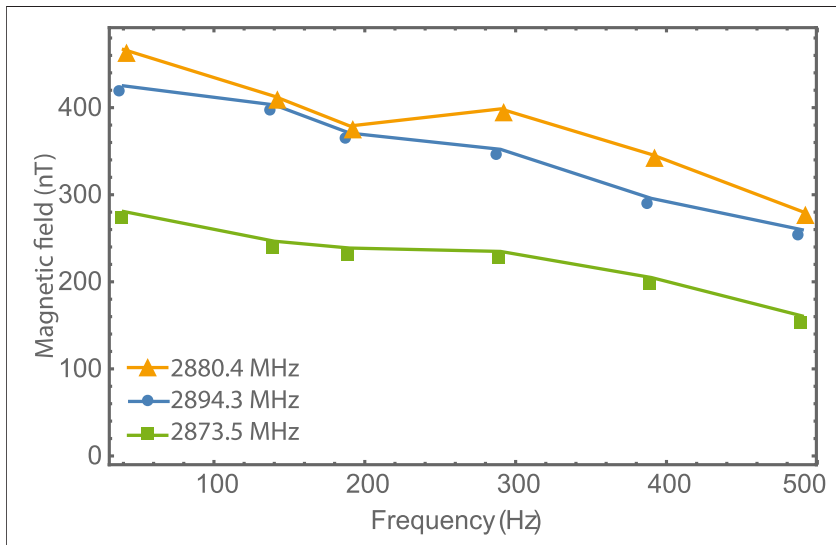

FIGURE 2 | Amplitude of magnetic test signal, measured along three different NV axes with the Bosch sensor. An oscillating, external magnetic field of about $0.7 \mu \mathrm{T}$ was applied to the sensor.

distance between the center of the diamond and the outer surface is roughly $2.4 \mathrm{~mm}$, the sensor can move independently as well. To filter low-frequency systematic noise components, e.g. laserpower fluctuations, the MW-frequency $\omega_{L}$ is modulated and the detected PL is demodulated with the LIA. To produce the magnetic field maps presented here, the Mainz sensor assembly was mounted on a computer-controlled motorized 3D translation stage (Thorlabs, MTS25/M-Z8) in the center of the Halbach array (not shown in Figure 1).

\section{MAGNETIC FIELD SENSITIVITY}

Figure 2 depicts the response of the Bosch sensor for three different NV axes upon application of an AC magnetic field with varying frequencies and a constant amplitude of $0.7 \mu \mathrm{T}$. The three 
TABLE 1 | Settings and detection limit for the three different NV axis used in the Bosch sensor.

\begin{tabular}{lccc}
\hline $\boldsymbol{f}_{\text {res }}$ & $\boldsymbol{f}_{\boldsymbol{m o d}}$ & $\boldsymbol{f}_{\text {depth }}$ & Detection limit \\
\hline $2,880.4 \mathrm{MHz}$ & $3 \mathrm{kHz}$ & $100 \mathrm{kHz}$ & $3.7 \pm 0.6 \mathrm{nT} / \sqrt{\mathrm{Hz}}$ \\
$2,894.3 \mathrm{MHz}$ & $2 \mathrm{kHz}$ & $100 \mathrm{kHz}$ & $3.1 \pm 0.3 \mathrm{nT} / \sqrt{\mathrm{Hz}}$ \\
$2,873.5 \mathrm{MHz}$ & $5 \mathrm{kHz}$ & $100 \mathrm{kHz}$ & $2.7 \pm 1.6 \mathrm{nT} / \sqrt{\mathrm{Hz}}$
\end{tabular}

axes see different effective amplitudes due to the different angles of the NV axes with respect to the magnetic field vector as expected. The sensor response slightly decreases with increasing frequency of the test field independent of the axis. This decrease might stem from the fact, that with increasing frequency, the excitation field becomes under-sampled meaning that the full reconstruction of the sinusoidal excitation field is not possible anymore leading to a decrease in measured amplitude. This decrease is not observable for the Mainz sensor, whose bandwidth is currently limited by the integration constant of the lock-in amplifier used during data acquisition.

To estimate the magnetic sensitivity of the two sensors we follow another method. When the MW field is resonant with the ground-state $m_{s}=0 \rightarrow m_{s}= \pm 1$ transitions, population is transferred through the excited triplet state to the metastable singlet state, resulting in PL reduction. PL as a function of MW frequency is the ODMR signal. By focusing around a single of feature in this ODMR signal the sensitivity can be extracted. The magnetic resonance for the Mainz sensor features a $350 \mathrm{kHz}$ linewidth and $1.6 \%$ contrast. The best sensitivity of the Bosch sensor was achieved with a linewidth of $\approx 92 \mathrm{kHz}$ and a contrast of $0.6 \%$. We modulate the MW frequency around a central frequency $\mathrm{f}_{c}$, and record the first harmonic of the transmission signal with a LIA. This generates a demodulated signal, which together with the $\mathrm{NV}$ gyromagnetic ratio of $|\gamma / 2 \pi| \sim$ 28.024 $\mathrm{GHz} \mathrm{T}^{-1}$ can be used to translate PL fluctuations to effective magnetic field noise. The optimized parameters resulting in the best magnetic field sensitivity for the Mainz sensor were: a modulation frequency of $\mathrm{f}_{\text {mod }}=13.6 \mathrm{kHz}$ and modulation amplitude of $\mathrm{f}_{a m p}=260 \mathrm{kHz}$. The sensitivity of the Bosch sensor was optimized for three different $\mathrm{NV}$ axes as summarized in Table $\mathbf{1}$.

Figure 3 shows the magnetic-field-noise spectrum of the sensors. The blue trace of Figure 3 corresponds to magnetically sensitive data of the Mainz sensor, the orange to magnetically insensitive. The magnetically sensitive data are taken at a MW frequency resonant with the NV electronic sublevels, while magnetically insensitive data are taken off resonance. The purple and green trace correspond to magnetically sensitive spectrum of the Bosch sensor outside and inside a magnetic shield, respectively, finally the red trace corresponds to the insensitive plot. The magnetically insensitive spectrum can be obtained if $\mathrm{f}_{c}$ is selected to be far from the ODMR features. The peak at $50 \mathrm{~Hz}$ is attributed to magnetic field from the power line in the lab. The average sensitivity in the $60-90 \mathrm{~Hz}$ area is $0.45 \mathrm{nT} / \sqrt{\mathrm{Hz}}$ and $0.34 \mathrm{nT} / \sqrt{\mathrm{Hz}}$, it is achieved for pump light power of 100 and $20 \mathrm{~mW}$, for the Mainz and Bosch sensors respectively.
The noise traces for the Bosch sensor are based on continuous data series, that are recorded with a sampling rate of $1 \mathrm{kHz}$ for $100 \mathrm{~s}$. To calculated the amplitude spectral density (ASD), the data series was split in 100 consecutive intervals, each with a duration of $1 \mathrm{~s}$. For each interval the ASD was calculated. The depicted is the average of the 100 ASDs.

\section{VECTOR MAGNETIC FIELD SENSING}

\subsection{Data Acquisition}

As a demonstration of the robustness and portability of our sensors as well as the ability to produce highly resolved magnetic field maps, we select the Mainz sensor to characterize the homogeneity of a custom-made Halbach-array magnet constructed in the Mainz laboratory. The schematic of the magnet is shown in Figure 4A with more details found in Ref. (Wickenbrock et al., 2020). It is a double ring of permanent magnets arranged to generate a homogeneous magnetic field in its inner bore along the radius of the rings. The field outside the construction decays rapidly with distance. We performed ODMR measurements in a $30 \times 20 \mathrm{~mm}^{2}$ plane nearly perpendicular to the main magnetic field direction in the center of the Halbach array in steps of 1 and $1.5 \mathrm{~mm}$ in $z$ and $y$ direction, respectively. The experimental procedure to characterize this magnet involves reconstruction of the $3 \mathrm{D}$ magnetic field from these ODMR measurements, which we describe in the next part of this paper. The measurements confirmed the homogeneity of the magnetic field of the magnet to be consistent with Hall-probe and NMR measurements, but with a threefold improved field strength resolution, vector information of the magnetic field and sub$\mathrm{mm}$ spatial information of these quantities. The orientation of the fiberized sensor in the Halbach magnet can be seen in Figure 4 a.

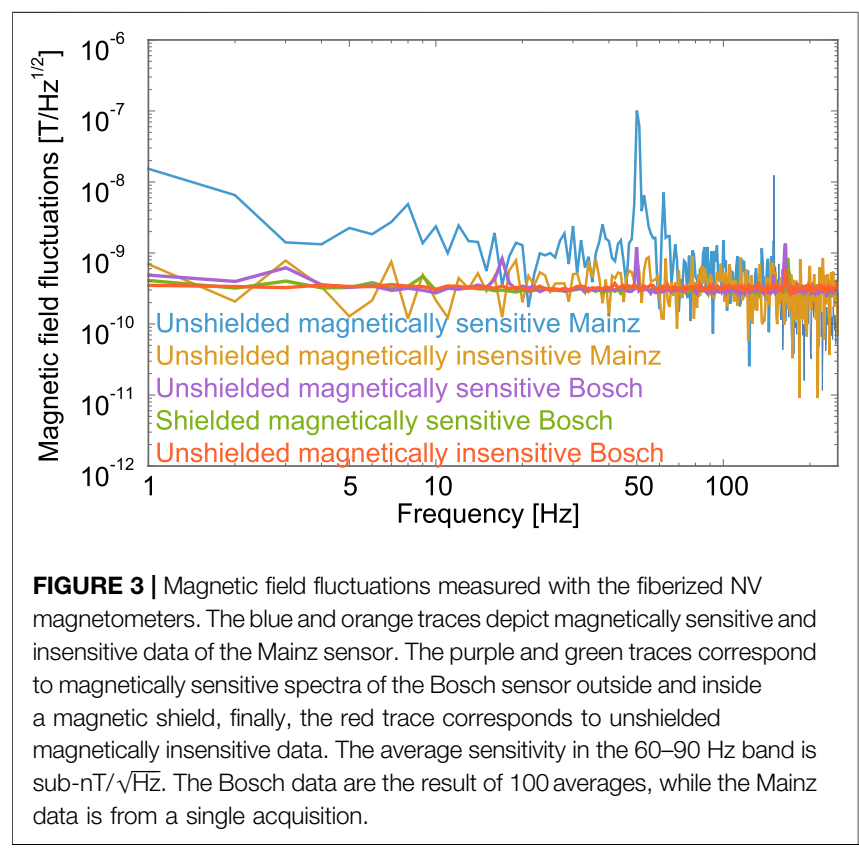


A

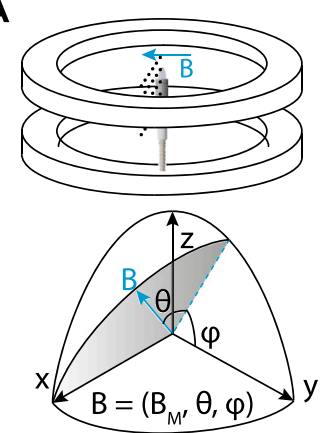

B

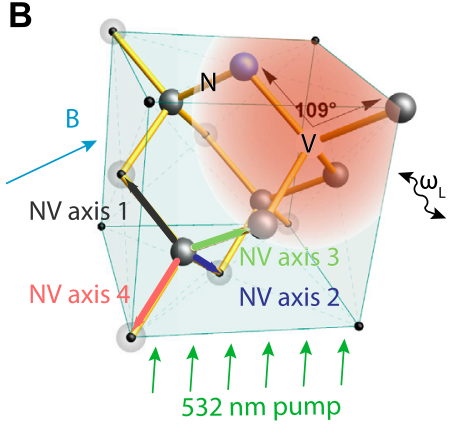

FIGURE 4 | (A) The orientation of the diamond sensor in the Halbach array. The orientation of the magnetic field is given in spherical coordinates with angle $\theta$ being the latitude angle with respect to the $x$-axis and $\phi$ the longitude. The main component of the magnetic field points along the $x$-axis. (B) lllustration of the diamond lattice structure indicating the four different NV axes 1,2,3,4 and their relation to the applied magnetic field $B$. Microwaves (MW) with frequency $\omega_{L} /(2 \pi)$ are applied to the sample.

The magnetic field is given in spherical coordinates with respect to the $(100)$ axis of the diamond $B=\left(B_{M}, \theta, \phi\right)$. The angle between the magnetic field vector and the yz-plane is $\theta$, and $\phi$ is the angle between the projection of $B$ in the yz-plane and the $y$-axis. The diamond sensor was oriented in the magnetic field such that different resonances were visible.

For each position in the $30 \times 20 \mathrm{~mm}^{2}$ plane a scan of the applied MW frequency between $3,800 \mathrm{MHz}$ and $5,000 \mathrm{MHz}$ was performed in steps of $1 \mathrm{MHz}$. This range was limited by the bandwidth of the MW components. At each frequency we recorded the demodulated PL from the LIA x-output with a data acquisition system and stored each data set with its respective position. To speed up the acquisition and after determining that no features were left out, we just acquired in

total 164 frequency values around the expected position of the resonances. In total a frequency scan to determine the four center frequencies lasted $46 \mathrm{~s}$. This can be dramatically improved by for example applying a frequency lock on the four transitions, respectively.

Figure 5 shows an example of collected data in four different points of the scan. The points are noted in Figure 8B with different colors. The four different frequency regions correspond to different kind of transitions as noted above the figures. The FWHM linewidth of the observed features is $(11.48 \pm 0.14) \mathrm{MHz}$ and therefore much larger than the one given above $(0.35 \pm 0.02)$ $\mathrm{MHz}$ for a small background field along one of the $\mathrm{NV}$ axis. This is due to the strong transverse field component but also caused intentional via MW power broadening. This simplified the lineshape by suppressing the hyperfine features in the spectrum and therefore the analysis routine. We additionally like to note, that the two first of the features are double quantum transitions (DQ), i.e. magnetic transitions from the $m_{s}=-1$ to the $m_{s}=+1$ state, normally forbidden, but allowed when transverse magnetic field components are present.

Overall the sensor was moved in steps of 1 and $1.5 \mathrm{~mm}$ in $z$ and $y$-direction, respectively, such that the whole area was covered. The resulting frequency maps of the chosen four resonances can be seen in Figure 8A; they show structures corresponding to the strength of the longitudinal (along the $\mathrm{NV}$ axis) and transverse (perpendicular to the NV axis) component of the magnetic field for a given diamond lattice axis. The information contained in these plots is more than sufficient to reconstruct all three vector components of the magnetic field at the position of the sensor.

\subsection{Frequency to Vector Field Conversion}

After acquiring MW frequency scans for different positions, the data were fitted with the sum of four derivatives of Lorentzians. The four center frequencies, four amplitudes and a combined

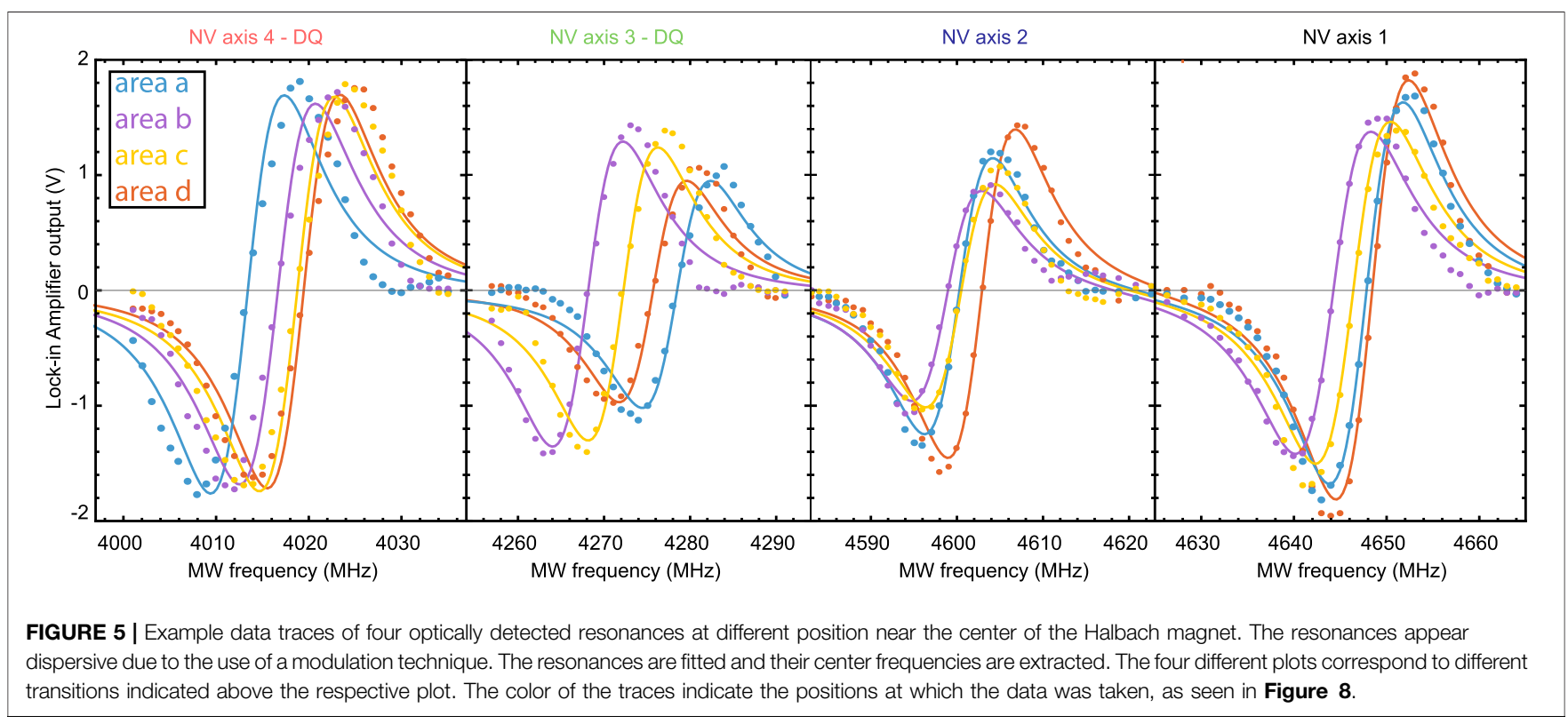




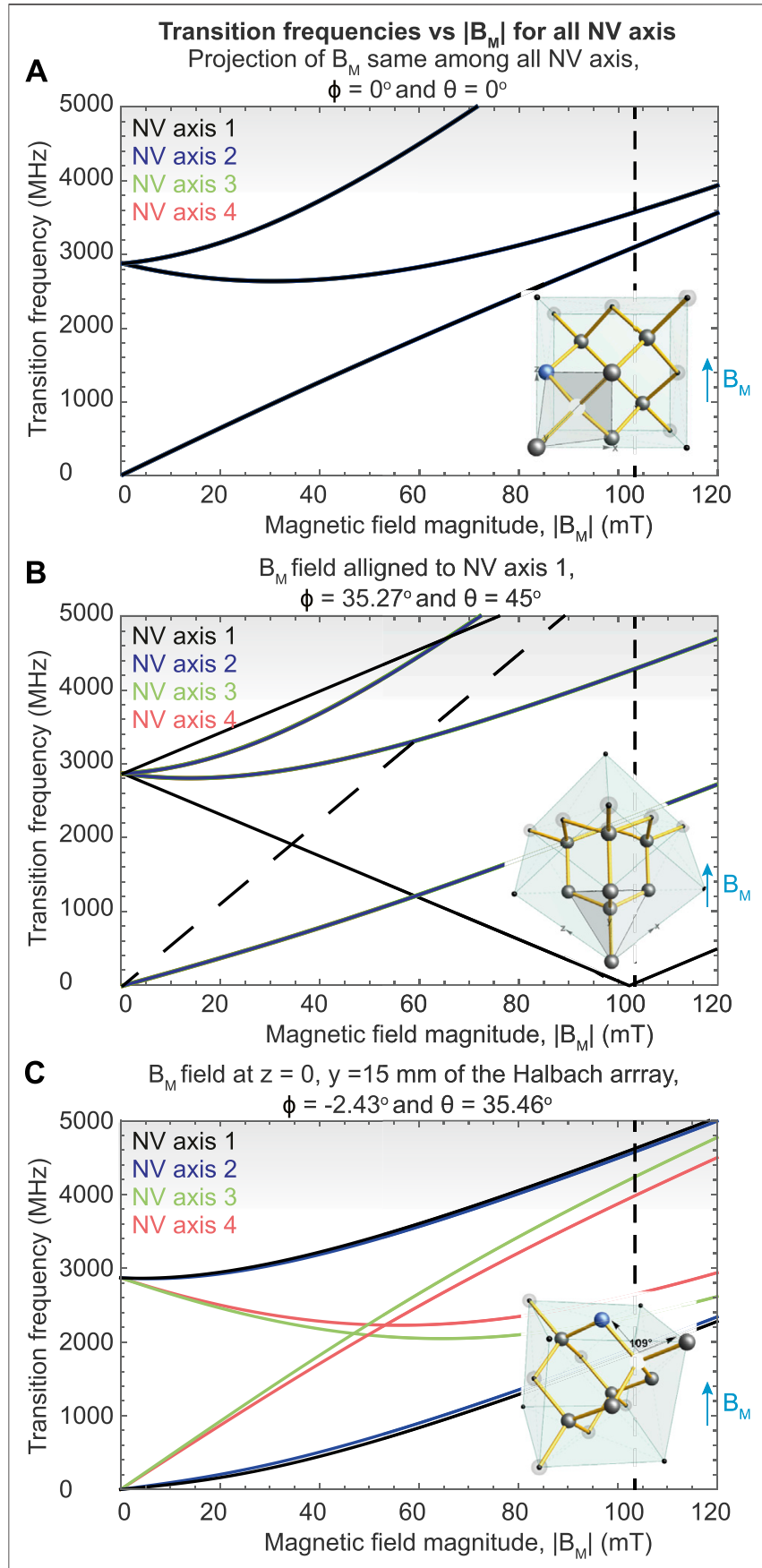

FIGURE 6 $\mid$ Transition frequencies as a function of $\left|B_{M}\right|$. The traces of the four different NV axes are depicted with colours consistent with those of Figure 4. The gray area represents our scanning range, the vertical dashed line shows the mean field strength inside the Halbach magnet. (A) For a magnetic field perpendicular to the (100) plane: all transitions overlap since the magnetic field projection is the same among all axes. (B) For a magnetic field perpendicular to the (111) plane of a diamond: the magnetic field is aligned along NV axis 1, and the magnetic field projection is the same for the other three axes. The slanted dashed line for NV axis one corresponds to the frequency of the $D Q$ transition which in this case due to the absence of transverse magnetic fields is not allowed. (C) For a magnetic field configuration inside the Halbach magnet.

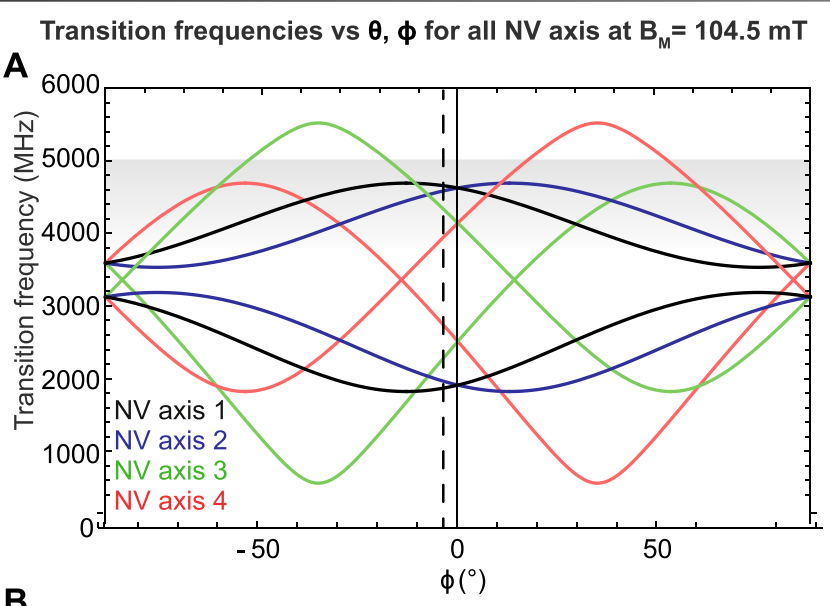

B

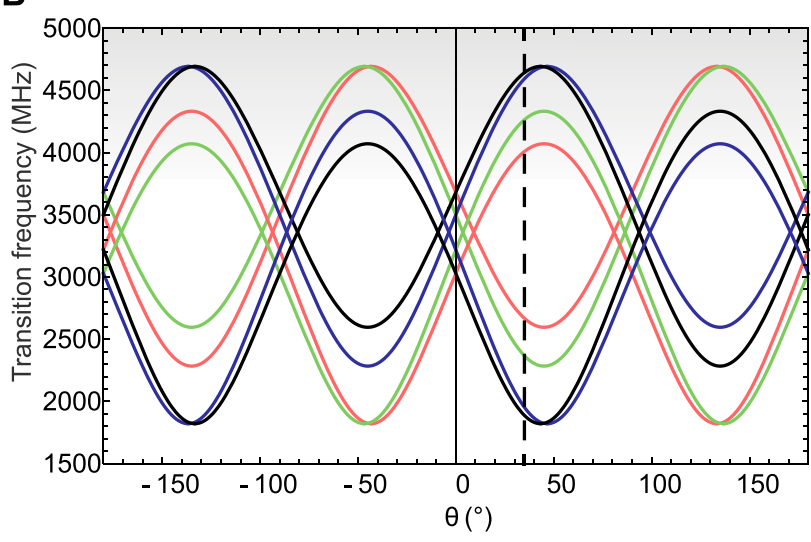

FIGURE 7 | Simulated transition frequencies as a function of angles $\theta$ and $\phi$ for the average magnetic field magnitude $\left|B_{M}\right|=104.5 \mathrm{mT}$ inside the Halbach magnet. The gray area represents our frequency scan range, the vertical dashed line indicates the respective average angle of all the measurements. (A) Transition frequencies as a function of angle $\theta$ for a fixed angle $\phi=-2^{\circ} 26^{\prime}$. (B) Transition frequencies as a function of angle $\phi$ for a fixed angle $\theta=35^{\circ} 27^{\prime}$

linewidth were fit parameters, an example of the data and fits can be seen in Figure 5. By matching these frequencies to the positions of the $3 \mathrm{D}$ translation stages we can make frequency maps as shown in Figure 8A.

To proceed with the analysis and construction of the magnetic field maps we note that the four different features presented in Figure 5 originate from the different NV orientations in the diamond crystal. The positions of these features are related to the alignment of the magnetic field to the NV axis, as well as its amplitude. If the magnetic field is along the NV axis (longitudinal) we observe the $m_{s}=0 \rightarrow m_{s}=$ \pm 1 transitions; if there is an additional magnetic field component orthogonal to the NV axis (transverse), transitions between the $m_{s}=$ -1 and $m_{s}=+1$ are allowed. We call these double quantum (DQ) transitions. For a given magnetic field direction at the position of the sensor, both, the longitudinal and transverse components of the magnetic field are present. Due to mixing caused by the transverse magnetic field component, the NV gyromagnetic ratio depends on the background field strength. 
A
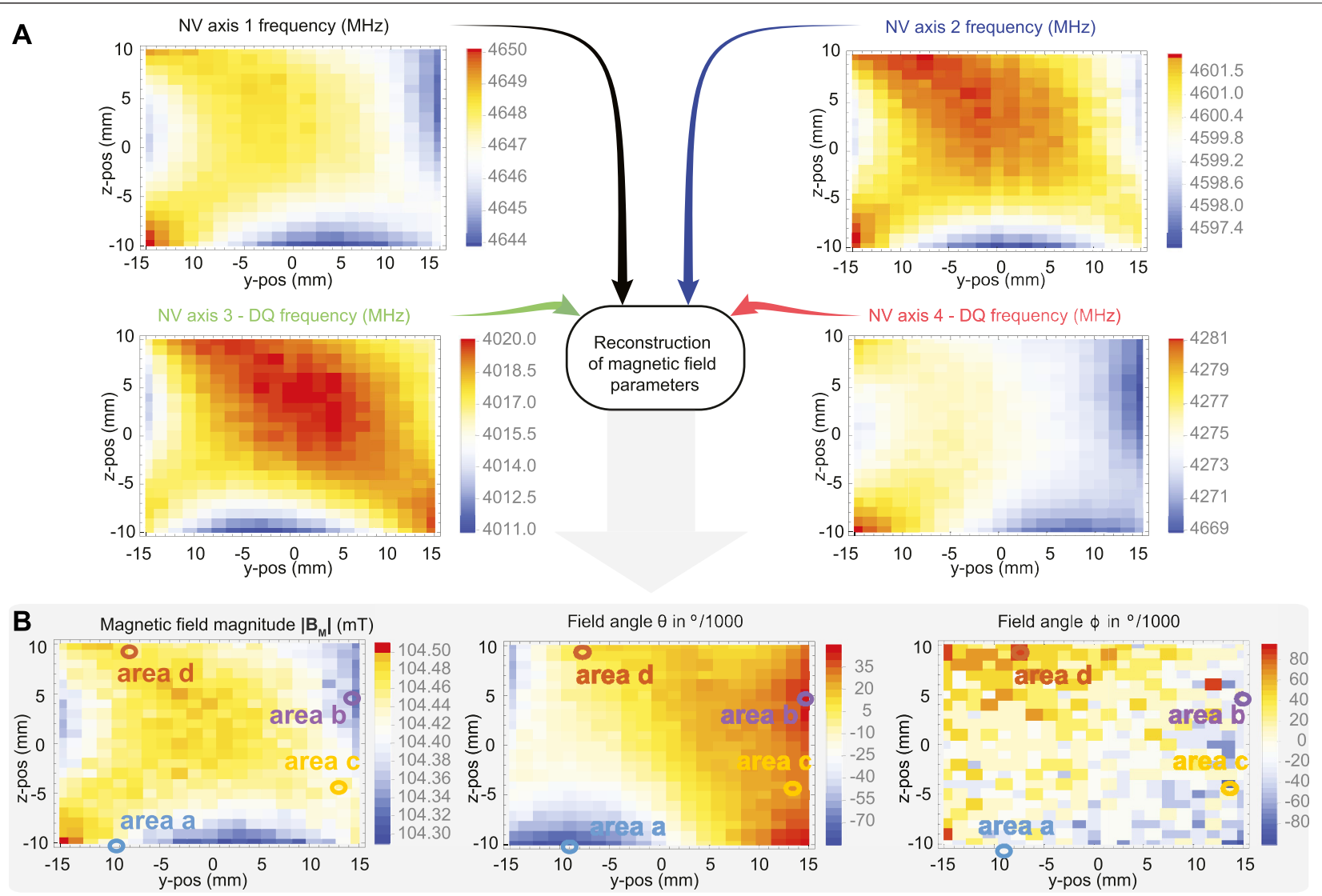

FIGURE 8| Figure adapted from Ref. (Wickenbrock et al., 2020). (A) Spatially resolved frequency maps of the individual resonances from Figure 5A. (B) The data collected are used to reconstruct the vector magnetic field in spherical coordinates as defined in Figure $\mathbf{4 A}$, the four colored circles note the positions at which data for Figure $\mathbf{5}$ are taken. Angles $\theta$ and $\phi$ are deviations from the mean value of the angles.

As a result, to match the above frequencies to magnetic fields we create four vectors describing the $\mathrm{NV}$ axes. We use parameters $\beta$ to describe the angle between NV axis and magnetic field and $\Omega$ for the field strength. With these parameters we derive a formula which describes the transition frequencies of the spin states, from $\mathrm{m}_{s}=0$ to $\mathrm{m}_{s}=-1$, as well as $\mathrm{m}_{s}=-1$ to $\mathrm{m}_{s}=+1$ (Rochester \& Budker, 2013). Here, we neglect strain and electric field effects.

The frequency of the resonances depends on the magnitube of the magnetic field $\mathrm{B}_{M}$, as well as the angles $\theta$ and $\phi$ of the diamond orientation with respect to the magnetic field. Figure 6 shows the resonance frequency dependence as a function of magnetic field magnitude for different fixed angles $\theta$ and $\phi$. Figures 6A,B represent a magnetic field perpendicular to common diamond surface cuts (100) plane for 1) and (111) plane for (b), 3) depicts a configuration inside our Halbach magnet. Figure 7 shows the dependence of the transition frequencies on the angles $\theta$ and $\phi$ for a magnetic field matching the mean magnitude inside the Halbach magnet. In Figure 7A $\phi=-2^{\circ} 26^{\prime}$ and $\theta$ is varied. In Figure 7B $\theta=35^{\circ} 27^{\prime}$ and $\phi$ is varied.

In both Figure 6 and Figure 7 the four different NV axes are depicted with colours matching those in Figure 4. We observe that in Figure 6A all the transitions overlap, as the projection of the magnetic field is the same among all axes. In Figure 6B the magnetic field is aligned along the NV axis 1 , and its projection is the same for the other three axes, which appear overlapped in the figure. The dashed line for NV axis one corresponds to the frequency of the DQ transition which in this case is not allowed, as there is no transverse field on $\mathrm{NV}$ axis 1. Figure 6C represents a case we encounter while measuring the field inside our Halbach magnet. In Figure 7 , we note the symmetry between $\mathrm{NV}$ axes 1,2 and 3,4 which arises because of the crystallographic structure of the diamond. We can use the information of the above plots to translate the frequency into magnetic field.

After identifying the possible transition frequencies, we use a numerical method to translate the frequencies into magnetic field direction and strength. For this method we restrict the magnetic field strength values to within $10 \%$ of what the estimated magnetic field produced by our Halbach array is. Once the magnetic field is calculated for all the different frequencies we express it in polar coordinates. When the initial values for direction and magnitude of the field are approximately known, we find that the reconstruction is unique. We choose $B_{M}$ to describe the strength of the field, angle $\theta$ the latitude angle with respect to the $x$-axis and $\phi$ the longitude angle as shown in Figure 4A. Finally, we make a list of these parameters and plot 
them as shown in Figure 8B. In Figure 8B we included four different coloured circles to represent the positions at which data for Figure 5 are taken. The measured averages of angles $\theta$ and $\phi$ in the central $10 \times 5 \mathrm{~mm}^{2}(\mathrm{y}-\mathrm{x})$ of the Halbach magnet are $-2^{\circ} 26 \pm$ $1^{\prime}$ and $35^{\circ} 2 \pm 1^{\prime}$, respectively, which is consistent with the orientation of the diamond lattice (cf. Figure 4) relative to the coordinate system of the magnet.

\section{CONCLUSION AND OUTLOOK}

We constructed two fiberized NV based magnetic field sensors. The sensors feature sub-nT/ $\sqrt{\mathrm{Hz}}$ magnetic field sensitivity with high PL-to-pump-light ratio. The components used to make the sensors are commercially available which makes their construction easy and reproducible. The design for the Mainz sensor was made in such a way as to allow free access to the front side of the diamond as well as for robustness, portability, and small size.

With one of the main challenges for NV magnetometry, especially fiberized, being the low photon-collection efficiency, which leads to poor photon-shot-noise limited sensitivity, the 0.5\% PL-to-pump-light ratio collection efficiency, demonstrated in the Mainz sensor, can be used to achieve even higher sensitivity, if the effect of other noise sources, e.g. laser-intensity, thermal and magnetic noise, is minimized. This ratio could also be further optimized by implementing side collection on the diamond(Sage et al., 2012; Barry et al., 2020), as well as highly reflective coatings on the opposite (front) side of the diamond. These features were not implemented in this sensor in order to keep all of its components commercial and easily accessible.

The access to the front side of the diamond allows for close proximity to magnetic field sources, which in turn leads to high spatial resolution. The proximity is currently limited to about $300 \mu \mathrm{m}$ by MW wire on top of the diamond. It could be optimized further by using a thinner diamond sample, a sample with shallow implanted NV centers or a thinner MW wire such as, for example, a capton-tape printed circuit board, all depending on the intended application, as these changes influence the sensitivity of the sensor. Such optimization would be especially beneficial for measurements of dipole fields or measurements that require high spatial resolution.

The robustness and portability of the sensors also make them attractive for mapping larger areas, or on-the-move measurements of

\section{REFERENCES}

Acosta, V. M., Bauch, E., Jarmola, A., Zipp, L. J., Ledbetter, M. P., and Budker, D. (2010). Broadband Magnetometry by Infrared-Absorption Detection of Nitrogen-Vacancy Ensembles in diamond. APL 97, 17. doi:10.1063/1.3507884 Balasubramanian, G., Chan, I. Y., Kolesov, R., Al-Hmoud, M., Tisler, J., Shin, C., et al. (2008). Nanoscale Imaging Magnetometry with diamond Spins under Ambient Conditions. Nature 455 (7213), 648-651. doi:10.1038/nature07278

Barry, J. F., Schloss, J. M., Bauch, E., Turner, M. J., Hart, C. A., Pham, L. M., et al. (2020). Sensitivity Optimization for Nv-diamond Magnetometry. Rev. Mod. Phys. 92, 015004. doi:10.1103/revmodphys.92.015004

Barry, J. F., Turner, M. J., Schloss, J. M., Glenn, D. R., Song, Y., Lukin, M. D., et al. (2016). Optical Magnetic Detection of Single-Neuron Action Potentials magnetic fields. These features combined with the small size of the sensors suggest a use for measuring in hard-to-access places or small areas where maneuverability of the sensor would be required, e.g. endoscopic measurements inside the human body.

As a demonstration of the Mainz sensor we measured the magnetic field of a custom-designed Halbach array. For these measurements, the small fiberized diamond sensor showed a $\mathrm{mm}$-scale spatial resolution, angular resolution of the magnetic field vector extracted in the measurements were $\times 910^{-3}$ degrees and $18 \times 10^{-3}$ degrees for angles $\theta$ and $\phi$, respectively. We note here, that the corresponding displacement due to such a rotation of the diamond sensor (considering a $(0.5 \times 0.5) \mathrm{mm}^{2}$ square cross section) could optically not be resolved.

\section{DATA AVAILABILITY STATEMENT}

The original contributions presented in the study are included in the article/supplementary material, further inquiries can be directed to the corresponding author/s.

\section{AUTHOR CONTRIBUTIONS}

All authors listed have made a substantial, direct, and intellectual contribution to the work and approved it for publication.

\section{FUNDING}

This work is supported by the EU FET-OPEN Flagship Project ASTERIQS (action 820394), the German Federal Ministry of Education and Research (BMBF) within the Quantumtechnologien program (grants FKZ 13N14439 and FKZ 13N15064), the Cluster of Excellence "Precision Physics, Fundamental Interactions, and Structure of Matter" (PRISMA + EXC 2118/1) funded by the German Research Foundation (DFG) within the German Excellence Strategy (Project ID 39083149).

\section{ACKNOWLEDGMENTS}

We acknowledge Fedor Jelezko for fruitful discussions.

Using Quantum Defects in diamond. PNAS 113, 14133. doi:10.1073/ pnas. 1601513113

Chatzidrosos, G., Wickenbrock, A., Bougas, L., Leefer, N., Wu, T., Jensen, K., et al. (2017). Miniature Cavity-Enhanced diamond Magnetometer. Phys. Rev. Appl. 8, 044019. doi:10.1103/physrevapplied.8.044019

Chatzidrosos, G., Wickenbrock, A., Bougas, L., Zheng, H., Tretiak, O., Yang, Y., et al. (2019). Eddy-current Imaging with Nitrogen-Vacancy Centers in diamond. Phys. Rev. Appl. 11, 014060. doi:10.1103/physrevapplied.11.014060

Clevenson, H., Pham, L. M., Teale, C., Johnson, K., Englund, D., and Braje, D. (2018). Robust High-Dynamic-Range Vector Magnetometry with Nitrogen-Vacancy Centers in diamond Featured. Appl. Phys. Lett. 112, 252406. doi:10.1063/1.5034216

Cochrane, C. J., Blacksberg, J., Anders, M. A., and Lenahan, P. M. (2016). Vectorized Magnetometer for Space Applications Using Electrical Readout of Atomic Scale Defects in Silicon Carbide. Scientific Rep. 6. doi:10.1038/srep37077 
Dumeige, Y., Chipaux, M., Jacques, V., Treussart, F., Roch, J.-F., Debuisschert, T., et al. (2013). Magnetometry with Nitrogen-Vacancy Ensembles in diamond Based on Infrared Absorption in a Doubly Resonant Optical Cavity. Phys. Rev. B 87, 155202. doi:10.1103/physrevb.87.155202

Dumeige, Y., Roch, J.-F., Bretenaker, F., Debuisschert, T., Acosta, V., Becher, C., et al. (2019). Infrared Laser Threshold Magnetometry with a Nv Doped diamond Intracavity Etalon. arXiv:2008.07339 27, 1706-1717. doi:10.1364/ oe.27.001706

Hadden, J. P., Harrison, J. P., Stanley-Clarke, A. C., Marseglia, L., Ho, Y.-L. D., Patton, B. R., et al. (2010). Strongly Enhanced Photon Collection from diamond Defect Centers under Microfabricated Integrated Solid Immersion Lenses. APL 97, 241901. doi:10.1063/1.3519847

Jensen, K., Leefer, N., Jarmola, A., Dumeige, Y., Acosta, V. M., Kehayias, P., et al. (2014). Cavity-enhanced Room-Temperature Magnetometry Using Absorption by Nitrogen-Vacancy Centers in diamond. Phys. Rev. Lett. 112, 160802. doi:10.1103/physrevlett.112.160802

Kucsko, G., Maurer, P. C., Yao, N. Y., Kubo, M., Noh, H. J., Lo, P. K., et al. (2013). Nanometre-scale Thermometry in a Living Cell. Nature 500, 54-58. doi:10.1038/nature12373

Lovchinsky, I., Sushkov, A. O., Urbach, E., Leon, N. P. d., Choi, S., Greve, K. D., et al. (2016). Nuclear Magnetic Resonance Detection and Spectroscopy of Single Proteins Using Quantum Logic. Science 351, 836. doi:10.1126/science.aad8022

Maze, J. R., Stanwix, P. L., Hodges, J. S., Hong, S., Taylor, J. M., Cappellaro, P., et al. (2008). Nanoscale Magnetic Sensing with an Individual Electronic Spin in diamond. Nature 455 (7213), 644-647. doi:10.1038/nature07279

Patel, R. L., Zhou, L. Q., Frangeskou, A. C., Stimpson, G. A., Breeze, B. G., Nikitin, A., et al. (2020). Sub-nanotesla Magnetometry with a FibreCoupled diamond Sensor. Phys. Rev. Appl. 14, 044058. doi:10.1103/ PhysRevApplied.14.044058

Rittweger, E., Han, K. Y., Irvine, S. E., Eggeling, C., and Hell, S. W. (2009). Sted Microscopy Reveals crystal Colour Centres with Nanometric Resolution. Nat. Photon. 3 (3), 144-147. doi:10.1038/nphoton.2009.2

Rochester, S., and Budker, D. (2013). NV Centers in diamond. online tutorial. Available at: http://budker.berkeley.edu/Tutorials/index.html.

Sage, D. L., Pham, L. M., Bar-Gill, N., Belthangady, C., Lukin, M. D., Yacoby, A., et al. (2012). Efficient Photon Detection from Color Centers in a diamond Optical Waveguide. Phys. Rev. B 85, 121202. doi:10.1103/physrevb.85.121202

Siyushev, P., Kaiser, F., Jacques, V., Gerhardt, I., Bischof, S., Fedder, H., et al. (2010). Monolithic diamond Optics for Single Photon Detection. APL 97, 241902. doi:10.1063/1.3519849

Stürner, F. M., Brenneis, A., Buck, T., Kassel, J., Rölver, R., Fuchs, T., et al. (2021). Integrated and Portable Magnetometer Based on Nitrogen-Vacancy Ensembles in diamond. Adv. Quantum Technol. 4 (4), 2000111. doi:10.1002/qute.202000111
Wickenbrock, A., Zheng, H., Bougas, L., Leefer, N., Afach, S., Jarmola, A., et al (2016). Microwave-free Magnetometry with Nitrogen-Vacancy Centers in diamond. Appl. Phys. Lett. 109, 053505. doi:10.1063/1.4960171

Wickenbrock, A., Zheng, H., Chatzidrosos, G., Rebeirro, J. S., Schneemann, T., and Bluemler, P. (2021). High Homogeneity Permanent Magnet for diamond Magnetometry. arXiv:2008.07339. 322, 106867. doi:10.1016/j.jmr.2020.106867

Wolf, T., Neumann, P., Nakamura, K., Sumiya, H., Ohshima, J., amd Isoya, T., et al. (2015). Subpicotesla diamond Magnetometry. Phys. Rev. X 5, 041001. doi:10.1103/physrevx.5.041001

Xie, Y., Yu, H., Zhu, Y., Qin, X., Rong, X., Duan, C.-K., et al. (2021). A Hybrid Magnetometer towards Femtotesla Sensitivity under Ambient Conditions. Sci. Bull. 66, 127. doi:10.1016/j.scib.2020.08.001

Zhang, C., Shagieva, F., Widmann, M., Kübler, F., Vorobyov, V., Kapitanova, P., et al. (2021). Diamond Magnetometry and Gradiometry towards Subpicotesla Dc Field Measurement. Phys. Rev. Appl. 15, 064075. doi:10.1103/physrevapplied.15.064075

Zheng, H., Chatzidrosos, G., Wickenbrock, A., Bougas, L., Lazda, R., Berzins, A., et al. (2017). Level Anti-crossing Magnetometry with Color Centers in diamond. Proc. SPIE, 10119, 101190X. doi:10.1117/12.2261160

Zheng, H., Sun, Z., Chatzidrosos, G., Zhang, C., Nakamura, K., Sumiya, H., et al. (2020). Microwave-free Vector Magnetometry with Nitrogen-Vacancy Centers along a Single axis in diamond. Phys. Rev. Appl. 13, 044023. doi:10.1103/ physrevapplied.13.044023

Conflict of Interest: Authors AB, FS, TF, TB, and RR were employed by the company Robert Bosch GmbH.

The remaining authors declare that the research was conducted in the absence of any commercial or financial relationships that could be construed as a potential conflict of interest.

Publisher's Note: All claims expressed in this article are solely those of the authors and do not necessarily represent those of their affiliated organizations, or those of the publisher, the editors and the reviewers. Any product that may be evaluated in this article, or claim that may be made by its manufacturer, is not guaranteed or endorsed by the publisher

Copyright (C) 2021 Chatzidrosos, Rebeirro, Zheng, Omar, Brenneis, Stürner, Fuchs, Buck, Rölver, Schneemann, Blümler, Budker and Wickenbrock. This is an openaccess article distributed under the terms of the Creative Commons Attribution License (CC BY). The use, distribution or reproduction in other forums is permitted, provided the original author(s) and the copyright owner(s) are credited and that the original publication in this journal is cited, in accordance with accepted academic practice. No use, distribution or reproduction is permitted which does not comply with these terms. 